\title{
ON A PROBLEM IN PLANE STRAIN
}

\author{
BY \\ H. J. GREENBERG AND ROHN TRUELL* \\ Brown University
}

1. Introduction. The present paper concerns the application to a particular problem in plane elasticity of the method recently developed by W. Prager and J. L. Synge for obtaining approximate solutions to boundary-value problems in elasticity.

The problem considered is that of an infinite bar of rectangular cross-section in a state of plane strain caused by compression as shown in Fig. 1. We assume the conditions of the test to be such that the bar is compressed by a prescribed amount $2 a$, the points on the top and bottom faces being permitted vertical $\left(u_{2}\right)$ but no horizontal $\left(u_{1}\right)$ displacement. Thus, after deformation the cross-section of the bar will appear as in Fig. 2.

This is not a simple compression test. In fact, the exact determination of the stresses

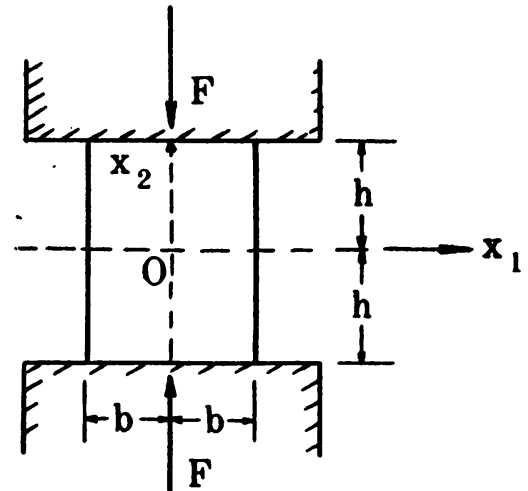

Fig. 1.

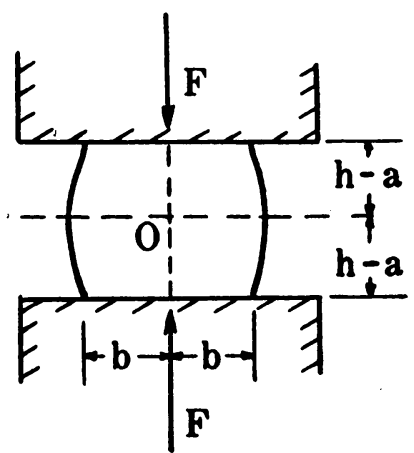

FIG. 2.

in the bar requires the solution of a mixed boundary value problem with the displacements prescribed along $x_{2}= \pm h$ and the stresses prescribed (to vanish) along $x_{1}= \pm b$.

We propose in the present paper to determine the relation between the force $F$ per unit length of the bar, the compression $2 a$ and the Young's modulus $E$ for the material assuming the value of Poisson's ratio $\nu$ to be known. (We note that the range of $\nu$ for most materials is small as compared with the possible range of $E$. In any case, by assuming extreme values of $\nu$ one may determine the effect of varying $\nu$ on the relation between $E, F$ and $a$.) Knowing this relation, the value of $E$ for a given material can be determined from test measurements of $F$ and $a$. This test has the advantage that the usual precautions taken to eliminate friction between specimen and end-blocks become unnecessary.

To solve the problem stated above one needs only to determine the strain energy of deformation of the bar. This we shall do to a good approximation by finding upper and lower bounds for this quantity. These bounds can be obtained by means of the Prager-Synge method. To apply this method we must construct artificial states of stress

*Received Aug. 30, 1947. This paper is based on a report prepared for Watertown Arsenal under a contract in Applied Mechanics.

${ }^{1}$ W. Prager and J. L. Synge, Quarterly Appl. Math. 5, 241-271 (1947). 
which approximate to the natural state of stress. These artificial states of stress will be discussed in the following section.

2. Specification of states of stress. According to the Prager-Synge method a state of stress, given by a set of six stress components $E_{i j}$ which are functions of position, is looked upon as defining a point or vector $\mathbf{S}$ in function space. Given two states $\mathbf{S}$ and $\mathbf{S}^{\prime}$, the scalar product of these states is defined to be

$$
\mathbf{S} \cdot \mathbf{S}^{\prime}=\int E_{i j} e_{i j}^{\prime} d v
$$

where $e_{i j}^{\prime}$ are the strains corresponding to the stresses $E_{i j}^{\prime}$ of the state $\mathbf{S}^{\prime}$ and are computed from the $E_{i j}^{\prime}$ by means of Hooke's law; the integral is extended over the volume of the body. It is easily verified that

$$
\mathbf{S} \cdot \mathbf{S}^{\prime}=\mathbf{S}^{\prime} \cdot \mathbf{S}
$$

so that the scalar product is commutative.

The sum $\mathbf{S}+\mathbf{S}^{\prime}$ of two states is defined as the state with the stresses $E_{i i}+E_{i j}^{\prime}$, the product $c \mathbf{S}$ of a state by a scalar $c$ is defined to be the state with the stresses $c E_{i j}$. It follows that the usual laws of vector algebra remain unchanged.

We shall reserve the notation $\mathbf{S}$ for the natural state of stress $E_{i j}$ in the bar. Thus, the stresses $E_{i j}$ are assumed to satisfy the equilibrium and compatibility equations and in addition all of the boundary conditions of the problem. We note that

$$
S^{2}=\mathbf{S} \cdot \mathbf{S}=\int E_{i j} e_{i j} d v
$$

so that the square of the magnitude of $\mathbf{S}$ is equal to twice the strain energy of deformation. of the bar.

According to the method, we must now select various artificial states of stress which satisfy some but not all of the conditions of the problem. We note first that our boundary conditions specify the displacements on the top and bottom of the bar and zero stresses along the sides. This makes our problem one of a type considered by Prager and Synge and designated by them as Displacement Boundary Condition (DBC). In accordance with their treatment of problems of the DBC type we shall need the following states:

(a) A state $\mathbf{S}^{*}$ called the completely associated state. The stresses $E_{i j}^{*}$ must satisfy the compatibility equation. The displacements defined by the $E_{i j}^{*}$ are required to satisfy the prescribed boundary conditions on displacements.

(b) A sequence of states $\mathbf{S}_{1}^{\prime}, \mathbf{S}_{2}^{\prime}, \cdots, \mathbf{S}_{p}^{\prime}$ called the homogeneous associated states. The stresses ${ }_{p} E_{i j}^{\prime}$ of the state $\mathbf{S}_{p}^{\prime}$ must satisfy the compatibility equations. The displacements defined by the ${ }_{p} E_{i j}^{\prime}$ are required to vanish wherever displacements are prescribed by the boundary conditions.

(c) A sequence of states $\mathbf{S}_{1}^{\prime \prime}, \mathbf{S}_{2}^{\prime \prime}, \ldots, \mathbf{S}_{a}^{\prime \prime}$ called the complementary states. The stresses ${ }_{a} E_{i j}^{\prime \prime}$ of the state $\mathbf{S}_{a}^{\prime \prime}$ must satisfy the equilibrium equations and in addition the boundary conditions on stresses.

Since the bar under compression is assumed to be in plane strain, the displacements $u_{1}$ and $u_{2}$ are functions of $x_{1}$ and $x_{2}$ alone and there is no displacement perpendicular to the $x_{1} x_{2}$-plane. The boundary conditions to be satisfied by the state $\mathbf{S}^{*}$ are therefore simply 


$$
\left.\begin{array}{l}
u_{1}^{*}\left(x_{1}, \pm h\right)=0 \\
u_{2}^{*}\left(x_{1},+h\right)=-a, \quad u_{2}^{*}\left(x_{1},-h\right)=a,
\end{array}\right\}
$$

where $u_{1}^{*}$ and $u_{2}^{*}$ are the displacements which give rise to the stresses $E_{i}^{*}$ of the state $\mathrm{S}^{*}$. Accordingly, the boundary conditions to be satisfied by the displacements ${ }_{p} u_{1}^{\prime}$ and ${ }_{p} u_{2}^{\prime}$ associated with the stresses ${ }_{p} E_{i j}^{\prime}$ of the state $\mathbf{S}_{p}^{\prime}$ are

$$
\left.\begin{array}{l}
{ }_{p} u_{1}^{\prime}\left(x_{1}, \pm h\right)=0 \\
{ }_{p} u_{2}^{\prime}\left(x_{1}, \pm h\right)=0 .
\end{array}\right\}
$$

It is simplest to obtain the associated states by beginning with displacement functions satisfying the boundary conditions, computing from these the strains and finally from the strains computing the stresses by means of Hooke's law. The stresses so obtained are of course automatically compatible.

In plane strain the stress components $E_{13}$ and $E_{23}$ are identically zero and

$$
E_{33}=\nu\left(E_{11}+E_{22}\right)
$$

where $\nu$ is Poisson's ratio. Since all quantities are independent of the variable $x_{3}$, the equilibrium equations take the form

$$
\begin{aligned}
& \frac{\partial E_{11}}{\partial x_{1}}+\frac{\partial E_{12}}{\partial x_{2}}=0 \\
& \frac{\partial E_{21}}{\partial x_{1}}+\frac{\partial E_{22}}{\partial x_{2}}=0 .
\end{aligned}
$$

Since there are no loads applied to the sides of the bar we have the following boundary conditions on the stresses

$$
E_{11}\left( \pm b, x_{2}\right)=E_{12}\left( \pm b, x_{2}\right)=0 .
$$

The stresses ${ }_{a} E_{i j}^{\prime \prime}$ of the state $S_{a}^{\prime \prime}$ are chosen to satisfy (2.5) and (2.6). Only the three functions ${ }_{a} E_{11}^{\prime \prime},{ }_{a} E_{12}^{\prime \prime}$ and ${ }_{a} E_{22}^{\prime \prime}$ need be chosen for each $q$, since ${ }_{a} E_{21}^{\prime \prime}={ }_{a} E_{12}^{\prime \prime}$ and we may take ${ }_{a} E_{13}^{\prime \prime}={ }_{a} E_{23}^{\prime \prime}=0$ and ${ }_{a} E_{33}^{\prime \prime}=\nu\left({ }_{a} E_{11}^{\prime \prime}+{ }_{a} E_{22}^{\prime \prime}\right)$ by virtue of the preceding remarks.

3. Upper and lower bounds for Young's modulus. Prager and Synge gave the following formula for upper and lower bounds on the quantity $S^{2}$ which is twice the strain energy of deformation of the natural state:

$$
\sum_{q=1}^{n}\left(\mathbf{S}^{*} \cdot \mathbf{I}_{a}^{\prime \prime}\right)^{2} \leq S^{2} \leq S^{*^{2}}-\sum_{p=1}^{m}\left(\mathbf{S}^{*} \cdot \mathbf{I}_{p}^{\prime}\right)^{2} ;
$$

the states $\mathbf{I}_{p}^{\prime}$ are obtained from the states $\mathbf{S}_{p}^{\prime}$ by orthonormalizing these latter states according to the requirement that

$$
\mathbf{I}_{p}^{\prime} \cdot \mathbf{I}_{r}^{\prime}=\left\{\begin{array}{lll}
1, & \text { if } & p=r \\
0, & \text { if } & p \neq r
\end{array}\right.
$$

and the states $\mathbf{I}_{a}^{\prime \prime}$ are similarly obtained from the states $\mathbf{S}_{a}^{\prime \prime}$. Since the strain energy of deformation is equal to the work done by the external forces we have

$$
S^{2}=2 F a
$$


or in terms of the average normal stress $\sigma=F / 2 b$ exerted on the bar by the end blocks,

$$
S^{2}=4 a b \sigma .
$$

Furthermore, from the definition of the scalar products involved, the conditions on the various states $\mathbf{S}^{*}, \mathbf{S}_{p}^{\prime}, \mathbf{S}_{a}^{\prime \prime}$ and the manner of orthonormalization of the states $\mathbf{S}_{p}^{\prime}$ and $\mathbf{S}_{a}^{\prime \prime}$ it follows that the upper and lower bounds in (3.1) are proportional to Young's modulus $E$ and to $a^{2}$. We can therefore write for (3.1), taking into account (3.2),

$$
L_{n} E a^{2} \leq 2 F \dot{a} \leq U_{m} E a^{2}
$$

where

$$
\begin{gathered}
L_{n}=\frac{1}{E a^{2}} \sum_{a=1}^{n}\left(\mathbf{S}^{*} \cdot \mathbf{I}_{a}^{\prime \prime}\right)^{2}, \\
U_{m}=\frac{1}{E a^{2}}\left[S^{*^{2}}-\sum_{p=1}^{m}\left(\mathbf{S}^{*} \cdot \mathbf{I}_{p}^{\prime}\right)^{2}\right] .
\end{gathered}
$$

From (3.4) we at once obtain

$$
\frac{2 F}{a U_{m}} \leq E \leq \frac{2 F}{a L_{n}}
$$

or in terms of the average stress $\sigma$ and the "average strain" $\epsilon=a / h$

$$
\frac{4 b}{h U_{m}} \frac{\sigma}{\epsilon} \leq E \leq \frac{4 b}{h L_{n}} \frac{\sigma}{\epsilon} .
$$

Thus, we obtain upper and lower bounds for Young's modulus in terms of $F$ and $a$. Alternatively if we are given $E$ and $F$ we can find upper and lower bounds for $a$ or given $E$ and $a$ we can find upper and lower bounds for $F$. Since $L_{n}$ increases and $U_{m}$ decreases as more terms are added in (3.5) (i.e., as $n$ and $m$ are increased) the bounds can be made to differ by a negligible amount so that in effect we determine the numerical coefficient $\lambda$ in the formula

$$
E=\lambda \frac{F}{a} .
$$

We note that the quantities $L_{n}$ and $U_{m}$ depend on Poisson's ratio $\nu$ which we must therefore assume to be known. The exact form of the dependence however can be easily obtained for $n=m=1$.

4. Selecting the functions and computing the bounds. ${ }^{2}$ For convenience we shall take $b=h=1$ and $\nu=\frac{1}{3}$ in the following. The completely associated state $\mathbf{S}^{*}$ and the five homogeneous associated states $\mathbf{S}_{\mathbf{1}}^{\prime}, \mathbf{S}_{\mathbf{2}}^{\prime}, \mathbf{S}_{\mathbf{3}}^{\prime}, \mathbf{S}_{\mathbf{4}}^{\prime}, \mathbf{S}_{5}^{\prime}$ which we use are given in Table 1. Tabulated are the displacement components, strains and stresses corresponding to each of these states. For convenience we have also set $E=1$ and $a=1$; these quantities are easily reintroduced into the final answers by proper proportionality factors.

The complementary states $\mathbf{S}_{p}^{\prime \prime}, p=1,2, \cdots$, are most conveniently defined by means of the Airy stress function $\psi$. The stresses are then computed from the function $\psi$ by taking

${ }^{2}$ In this work we were ably assisted by Mr. F. Edelman who performed the bulk of the computations. 


$$
\left.\begin{array}{l}
E_{11}^{\prime \prime}=\frac{\partial^{2} \psi}{\partial x_{2}^{2}} \\
E_{22}^{\prime \prime}=\frac{\partial^{2} \psi}{\partial x_{1}^{2}} \\
E_{12}^{\prime \prime}=-\frac{\partial^{2} \psi}{\partial x_{1} \partial x_{2}}
\end{array}\right\}
$$

When defined in this way, the stresses automatically satisfy the equilibrium equations (2.5) for any choice of the function $\psi$. We note that not any function $\psi$ may be used, however, since the stresses must satisfy the boundary conditions (2.6) where $b=1$. The five complementary states we use are given in Table 2.

In orthonormalizing the sequence of states $\mathbf{S}_{\mathbf{1}}^{\prime}, \cdots, \mathbf{S}_{\mathbf{5}}^{\prime}$ to obtain the orthonormal sequence $\mathbf{I}_{1}^{\prime}, \cdots, \mathbf{I}_{s}^{\prime}$ the scalar products $\mathbf{S}_{i}^{\prime} \cdot \mathbf{S}_{i}^{\prime}$ are required. These are listed in Table 3. To illustrate, we have $S_{2}^{\prime} \cdot S_{2}^{\prime}=4.8, S_{3}^{\prime} \cdot S_{5}^{\prime}=S_{5}^{\prime} \cdot S_{3}^{\prime}=0.925714$, etc. Also given in Table 3 are the values of the scalar products $\mathbf{S}^{*} \cdot \mathbf{S}_{i}^{\prime}, j=1, \cdots, 5$ and $\mathbf{S}^{*} \cdot \mathbf{S}^{*}$ which

\begin{tabular}{|c|c|c|c|c|c|}
\hline State & $u_{1}^{\prime}$ & $u_{2}^{\prime}$ & $e_{11}^{\prime}=\frac{\partial u_{1}^{\prime}}{\partial x_{1}}$ & $e_{12}^{\prime}=\frac{1}{2}\left(\frac{\partial u_{1}^{\prime}}{\partial x_{2}}+\frac{\partial u_{2}^{\prime}}{\partial x_{1}}\right)$ & $e_{22}^{\prime}=\frac{\partial u_{2}^{\prime}}{\partial x_{2}}$ \\
\hline $\mathbf{S}_{1}^{\prime}$ & $x_{1}\left(1-x_{2}^{2}\right)$ & 0 & $1-x_{2}^{2}$ & $-x_{1} x_{2}$ & 0 \\
\hline $\mathbf{S}_{2}^{\prime}$ & 0 & $-x_{2}\left(1-x_{2}^{2}\right)$ & 0 & 0 & $3 x_{2}^{2}-1$ \\
\hline $\mathbf{S}_{3}^{\prime}$ & $x_{1}^{3}\left(1-x_{2}^{2}\right)$ & 0 & $3 x_{1}^{2}\left(1-x_{2}^{2}\right)$ & $-x_{1} x_{2}^{3}$ & 0 \\
\hline $\mathbf{S}_{4}^{\prime}$ & 0 & $-x_{2}\left(1-x_{2}^{2}\right) x_{1}^{2}$ & 0 & $-x_{1} x_{2}\left(1-x_{2}^{2}\right)$ & $x_{1}^{2}\left(3 x_{2}^{2}-1\right)$ \\
\hline $\mathbf{S}_{s}^{\prime}$ & $x_{1}^{3} x_{2}^{2}\left(1-x_{2}^{2}\right)$ & 0 & $3 x_{1}^{2} x_{2}^{2}\left(1-x_{2}^{2}\right)$ & $x_{1}^{3} x_{2}\left(1-2 x_{2}^{2}\right)$ & 0 \\
\hline $\mathbf{S}^{*}$ & $u_{1}^{*}=0$ & $u_{2}^{*}=-x_{2}$ & $e_{11}^{*}=0$ & $e_{12}^{*}=0$ & $e_{22}^{*}=-1$ \\
\hline
\end{tabular}
are needed in the evaluation of the quantities in (3.5).

\begin{tabular}{|c|c|c|c|}
\hline State & $E_{11}^{\prime}=\frac{3}{4}\left(2 e_{11}^{\prime}+e_{22}^{\prime}\right)$ & $E_{12}^{\prime}=\frac{3}{4} e_{12}^{\prime}$ & $E_{22}^{\prime}=\frac{3}{4}\left(2 e_{22}^{\prime}+e_{11}^{\prime}\right)$ \\
\hline $\mathbf{S}_{1}^{\prime}$ & $\frac{3}{2}\left(1-x_{2}^{2}\right)$ & $-\frac{3}{4} x_{1} x_{2}$ & $\frac{3}{4}\left(1-x_{2}^{2}\right)$ \\
\hline $\mathbf{S}_{2}^{\prime}$ & $\frac{3}{4}\left(3 x_{2}^{2}-1\right)$ & 0 & $\frac{3}{2}\left(3 x_{2}^{2}-1\right)$ \\
\hline $\mathbf{S}_{3}^{\prime}$ & $\frac{9}{2} x_{1}^{2}\left(1-x_{2}^{2}\right)$ & $-\frac{3}{4} x_{1} x_{2}^{3}$ & $\frac{9}{4} x_{1}^{2}\left(1-x_{2}^{2}\right)$ \\
\hline $\mathbf{S}_{4}^{\prime}$ & $\frac{3}{4} x_{1}^{2}\left(3 x_{2}^{2}-1\right)$ & $-\frac{3}{4} x_{1} x_{2}\left(1-x_{2}^{2}\right)$ & $\frac{3}{2} x_{1}^{2}\left(3 x_{2}^{2}-1\right)$ \\
\hline $\mathbf{S}_{\mathbf{s}}^{\prime}$ & $\frac{9}{2} x_{1}^{2} x_{2}^{2}\left(1-x_{2}^{2}\right)$ & $\frac{3}{4} x_{1}^{3} x_{2}\left(1-2 x_{2}^{2}\right)$ & $\frac{9}{4} x_{1}^{2} x_{2}^{2}\left(1-x_{2}^{2}\right)$ \\
\hline $\mathbf{S}^{*}$ & $E_{11}^{*}=-\frac{3}{4}$ & $E_{12}^{*}=0$ & $E_{22}^{*}=-\frac{3}{2}$ \\
\hline
\end{tabular}

TABLE 1: The associated states. 
TABLE 2: The complementary states.

\begin{tabular}{|c|c|c|c|c|c|}
\hline State & $\psi$ & $E_{11}^{\prime \prime}=\frac{\partial^{2} \psi}{\partial x_{2}^{2}}$ & \multicolumn{2}{|c|}{$E_{12}^{\prime \prime}=-\frac{\partial^{2} \psi}{\partial x_{1} \partial x_{2}}$} & $E_{22}^{\prime \prime}=\frac{\partial^{2} \psi}{\partial x_{1}^{2}}$ \\
\hline $\mathbf{S}_{\mathbf{1}}^{\prime \prime}$ & $-\frac{1}{2} x_{1}^{2}$ & 0 & \multicolumn{2}{|l|}{0} & -1 \\
\hline $\mathbf{S}_{2}^{\prime \prime}$ & $-\frac{1}{12} x_{1}^{4}$ & 0 & \multicolumn{2}{|l|}{0} & $-x_{1}^{2}$ \\
\hline $\mathbf{S}_{3}^{\prime \prime}$ & $\frac{1}{2} x_{2}^{2}\left(1-x_{1}^{2}\right)^{2}$ & $\left(1-x_{1}^{2}\right)^{2}$ & \multicolumn{2}{|l|}{$4 x_{1} x_{2}\left(1-x_{1}^{2}\right)$} & $-2 x_{2}^{2}\left(1-3 x_{1}^{2}\right)$ \\
\hline $\mathbf{S}_{4}^{\prime \prime}$ & $\frac{1}{4} x_{2}^{4}\left(1-x_{1}^{2}\right)^{2}$ & $3 x_{2}^{2}\left(1-x_{1}^{2}\right)^{2}$ & \multicolumn{2}{|l|}{$4 x_{1} x_{2}^{3}\left(1-x_{1}^{2}\right)$} & $-x_{2}^{4}\left(1-3 x_{1}^{2}\right)$ \\
\hline $\mathbf{S}_{s}^{\prime \prime}$ & $\frac{1}{2} x_{1}^{2} x_{2}^{2}\left(1-x_{1}^{2}\right)^{2}$ & $x_{1}^{2}\left(1-x_{1}^{2}\right)^{2}$ & \multicolumn{2}{|c|}{$-2 x_{2}\left(3 x_{1}^{5}-4 x_{1}^{3}+x_{1}\right)$} & $x_{2}^{2}\left(15 x_{1}^{4}-12 x_{1}^{2}+1\right)$ \\
\hline State & \multicolumn{2}{|c|}{$e_{11}^{\prime \prime}=\frac{4}{9}\left[2 E_{11}^{\prime \prime}-E_{22}^{\prime \prime}\right]$} & $e_{12}^{\prime \prime}=\frac{4}{3} E_{12}^{\prime \prime}$ & \multicolumn{2}{|c|}{$e_{22}^{\prime \prime}=\frac{4}{9}\left[2 E_{22}^{\prime \prime}-E_{11}^{\prime \prime}\right]$} \\
\hline $\mathbf{S}_{\mathbf{1}}^{\prime \prime}$ & \multicolumn{2}{|c|}{$\frac{4}{9}$} & 0 & & $-\frac{8}{9}$ \\
\hline $\mathbf{S}_{2}^{\prime \prime}$ & \multicolumn{2}{|l|}{$\frac{4}{9} x_{1}^{2}$} & 0 & & $-\frac{8}{9} x_{1}^{2}$ \\
\hline $\mathbf{S}_{3}^{\prime \prime}$ & \multicolumn{2}{|c|}{$\frac{8}{9}\left[\left(1-x_{1}^{2}\right)^{2}+x_{2}^{2}\left(1-3 x_{1}^{2}\right)\right]$} & $\frac{16}{3} x_{1} x_{2}\left(1-x_{1}^{2}\right)$ & \multicolumn{2}{|c|}{$-\frac{4}{9}\left[4 x_{2}^{2}\left(1-3 x_{1}^{2}\right)+\left(1-x_{1}^{2}\right)^{2}\right]$} \\
\hline$\cdot$ & \multicolumn{2}{|c|}{$\frac{4}{9}\left[6 x_{2}^{2}\left(1-x_{1}^{2}\right)^{2}+x_{2}^{4}(1\right.$} & $\frac{16}{3} x_{1} x_{2}^{3}\left(1-x_{1}^{2}\right)$ & \multicolumn{2}{|c|}{$-\frac{4}{9}\left[2 x_{2}^{4}\left(1-3 x_{1}^{2}\right)+3 x_{2}^{2}(1\right.$} \\
\hline $\mathbf{S}_{s}^{\prime \prime}$ & \multicolumn{2}{|c|}{$\frac{4}{9}\left[2 x_{1}^{2}\left(1-x_{1}^{2}\right)^{2}-x_{2}^{2}\left(15 x_{1}^{4}\right.\right.$} & $\begin{array}{l}-\frac{8}{3} x_{2}\left(3 x_{1}^{5}\right. \\
\left.-4 x_{1}^{3}+x_{1}\right)\end{array}$ & \multicolumn{2}{|c|}{$\begin{array}{c}\frac{4}{9}\left[2 x_{2}^{2}\left(15 x_{1}^{4}-12 x_{1}^{2}+1\right)\right. \\
\left.-x_{1}^{2}\left(1-x_{1}^{2}\right)^{2}\right]\end{array}$} \\
\hline
\end{tabular}

TABLE 3: Values of $\mathbf{S}_{i}^{\prime} \cdot \mathbf{S}_{i}^{\prime}$ and $\mathbf{S}^{*} \cdot \mathbf{S}_{i}^{\prime}$.

\begin{tabular}{c||c|c|c|c|c|c}
\hline & $\mathrm{S}_{1}^{\prime}$ & $\mathrm{S}_{2}^{\prime}$ & $\mathrm{S}_{3}^{\prime}$ & $\mathrm{S}_{4}^{\prime}$ & $\mathrm{S}_{8}^{\prime}$ & $\mathrm{S}^{*}$ \\
\hline \hline $\mathrm{S}_{1}^{\prime}$ & 3.866667 & -0.8 & 3.6 & 0 & 0.537143 & -2 \\
\hline $\mathrm{S}_{2}^{\prime}$ & -0.8 & 4.8 & -0.8 & 1.6 & 0.114286 & 0 \\
\hline $\mathrm{S}_{3}^{\prime}$ & 3.6 & -0.8 & 6.045714 & -.365714 & 0.925714 & -2. \\
\hline $\mathrm{S}_{4}^{\prime}$ & 0 & 1.6 & -.365714 & 1.112381 & 0.045714 & 0 \\
\hline $\mathrm{S}_{5}^{\prime}$ & 0.537143 & 0.114286 & 0.925714 & 0.045714 & .364080 & -0.4 \\
\hline $\mathrm{S}^{*}$ & -2 & 0 & -2 & 0 & -0.4 & 6. \\
\hline
\end{tabular}


TABLE 4: Values of $r_{i p}^{\prime}=a_{i p} / a_{p p}$ and of $a_{p p}^{2}$.

\begin{tabular}{r||c|c|c|c|c|c}
\hline & $p=1$ & 2 & 3 & 4 & 5 & $a_{p p}^{2}$ \\
\hline \hline$i=1$ & 1. & 0.206897 & -0.928571 & -0.190946 & 0.007212 & 0.258621 \\
\hline 2 & - & 1. & 0.011905 & -0.343706 & -0.035183 & 0.215774 \\
\hline 3 & - & - & 1. & 0.128711 & -0.164771 & 0.371287 \\
\hline 4 & - & - & - & 1. & -0.044662 & 1.940316 \\
\hline 5 & - & - & - & - & 1. & 4.776450 \\
\hline
\end{tabular}

TABLE 5: Values of $\mathbf{S}_{i}^{\prime \prime} \cdot \mathbf{S}_{i}^{\prime \prime}$ and $\mathbf{S}^{*} \cdot \mathbf{S}_{i}^{\prime \prime}$.

\begin{tabular}{c||c|c|c|c|c|c}
\hline & $\mathrm{S}_{1}^{\prime \prime}$ & \multicolumn{1}{c|}{$\mathrm{S}_{2}^{\prime \prime}$} & \multicolumn{1}{c|}{$\mathrm{S}_{3}^{\prime \prime}$} & \multicolumn{1}{c|}{$\mathrm{S}_{4}^{\prime \prime}$} & \multicolumn{1}{c|}{$\mathrm{S}_{5}^{\prime \prime}$} & $\mathrm{S}^{*}$ \\
\hline \hline $\mathrm{S}_{1}^{\prime \prime}$ & 3.555556 & 1.185185 & 0.948148 & 0.948148 & 0.135450 & 4. \\
\hline $\mathrm{S}_{2}^{\prime \prime}$ & 1.185185 & 0.711111 & -0.496649 & -0.054180 & -0.045150 & 1.333333 \\
\hline $\mathrm{S}_{3}^{\prime \prime}$ & 0.948148 & -0.496649 & 8.777141 & 5.616633 & 0.456424 & 0 \\
\hline $\mathrm{S}_{4}^{\prime \prime}$ & 0.948148 & -0.054180 & 5.616633 & 5.238667 & 0.247434 & 0 \\
\hline $\mathrm{S}_{5}^{\prime \prime}$ & 0.135450 & -0.045150 & 0.456424 & 0.247434 & 1.465237 & 0 \\
\hline $\mathrm{S}^{*}$ & 4 & 1.333333 & 0 & 0 & 0 & 6 \\
\hline
\end{tabular}

TABLE 6: Values of $r_{i p}^{\prime \prime}=b_{i p} / b_{p p}$ and of $b_{p p}^{2}$.

\begin{tabular}{r||c|c|c|c|c|c}
\hline & $p=1$ & 2 & 3 & 4 & 5 & $b_{p p}^{2}$ \\
\hline \hline$i=1$ & 1 & -0.333333 & -1.123812 & 0.113388 & -0.098739 & 0.281250 \\
\hline 2 & - & 1. & 2.571430 & -0.591654 & 0.201460 & 3.164056 \\
\hline 3 & - & - & 1. & -0.685644 & -0.039757 & 0.155412 \\
\hline 4 & - & - & - & 1. & 0.015344 & 0.654785 \\
\hline 5 & - & - & - & - & 1. & 0.700065 \\
\hline
\end{tabular}

Writing

$$
\mathbf{I}_{p}^{\prime}=\sum_{i=1}^{p} a_{i p} \mathbf{S}_{i}^{\prime}, \quad p=1, \cdots, 5
$$

where the constants $a_{i p}$ are to be determined according to the usual orthogonalization scheme, we have for $U_{m}$ in (3.5)

$$
U_{m}=\frac{1}{E a^{2}}\left[S^{*^{2}}-\sum_{p=1}^{m}\left(\mathbf{S}^{*} \cdot \sum_{i=1}^{p} a_{i p} \mathbf{S}_{i}^{\prime}\right)^{2}\right], \quad-m=1, \cdots, 5 .
$$

In expanding, only products $a_{i p} a_{i p}(i, j=1 \cdots, p$ where $p=1, \cdots, 5)$ of the co- 
efficients enter. Defining $r_{i p}^{\prime}=a_{i p} / a_{p p},(i=1, \cdots, p$ for a fixed $p=1, \cdots, 5)$ we have

$$
a_{i p} a_{i p}=r_{i p}^{\prime} r_{i p}^{\prime} a_{p p}^{2}
$$

where the $r_{i p}^{\prime}$ are obtained for each $p$ as solutions of the $(p-1)$ linear equations

$$
\sum_{i=1}^{p-1}\left(\mathbf{S}_{i}^{\prime} \cdot \mathbf{S}_{i}^{\prime}\right) r_{i p}^{\prime}=-\mathbf{S}_{i}^{\prime} \cdot \mathbf{S}_{p}^{\prime}, \quad j=1, \cdots, p-1
$$

whose coefficients are the products $\mathbf{S}_{i}^{\prime} \cdot \mathbf{S}_{i}^{\prime}$ of Table 3 . The values of the $r_{i p}^{\prime}$ are given in Table 4 as well as the values of the quantities $a_{11}^{2}, a_{22}^{2}, \cdots, a_{55}^{2}$. From these values and the values of the $\mathbf{S}^{*} \cdot \mathbf{S}_{j}^{\prime}$ in Table 3 we may compute $U_{1}, \cdots, U_{5}$ from (4.3).

Similarly, Table 5 gives the values of the scalar products of the complementary states with one another and with $\mathbf{S}^{*}$. Writing

$$
\mathbf{I}_{a}^{\prime \prime}=\sum_{j=1}^{q} b_{i q} \mathbf{S}_{i}^{\prime \prime}
$$

we get for (3.5)

$$
L_{n}=\frac{1}{E a^{2}} \sum_{a=1}^{n}\left(\mathbf{S}^{*} \cdot \sum_{i=1}^{q} b_{i a} \mathbf{S}_{i}^{\prime \prime}\right)^{2}, \quad n=1, \cdots, 5
$$

The products of the coefficients are given by

$$
b_{i q} b_{i q}=r_{i q}^{\prime \prime} r_{i q}^{\prime \prime} b_{a q}^{2}, \quad i, j=1, \cdots, q \quad q=1, \cdots, 5
$$

where the values of the $r_{i a}^{\prime \prime}$ and the $b_{a e}^{2}$ are to be found in Table 6 .

Evaluating (4.3) and (4.4) we find (reentering the factors $E$ and $a$ so that the quantity $E a^{2}$ cancels out) the values of $L_{1}, \cdots, L_{3}$ and $U_{1}, \cdots, U_{5}$ given in Table 7 below.

TABLE 7

\begin{tabular}{c||c|cc}
\hline & $L_{n}$ & $U_{m}$ & \\
\hline \hline$m=n=1$ & 4.500000 & 4.965516 & (5 places accurate, 6'th estimated) \\
\cline { 2 - 3 } 2 & 4.500000 & 4.928572 & \\
\hline 3 & 4.676823 & 4.920992 \\
\hline 4 & 4.750435 & 4.890932 & \\
\hline 5 & 4.761603 & 4.856468 & (4 places accurate) \\
\hline
\end{tabular}

Entering these values in (3.6) we get successively improved inequalities for $E$ as follows 
TABLE 8

\begin{tabular}{c|c}
\hline $\begin{array}{c}\text { Iteration } \\
\text { No. }\end{array}$ & \\
\hline 1 & $.402778 \frac{F}{a} \leq E \leq .444444 \frac{F}{a}$ \\
2 & $.405797 \frac{F}{a} \leq E \leq .444444 \frac{F}{a}$ \\
3 & $.406422 \frac{F}{a} \leq E \leq .427641 \frac{F}{a}$ \\
4 & $.408920 \frac{F}{a} \leq E \leq .421014 \frac{F}{a}$ \\
5 & $.411822 \frac{F}{a} \leq E \leq .420027 \frac{F}{a}$
\end{tabular}

At the first iteration the gap between upper and lower bound is $10.3 \%$. In four further iterations this is reduced to $1.99 \%$. Averaging the upper and lower bounds yielded at the fifth iteration and retaining only 4 places gives the approximate formula

$$
E=.4159 \frac{F}{a}
$$

where we have established that the coefficient is in error by less than $1 \%$. The corresponding expression in terms of average stress and strain is

$$
E=.8318 \frac{\sigma}{\epsilon} .
$$

It is interesting to compare these formulas with those for a test in which motion of the bar in the $x_{1}$-direction is not hindered, i.e. with the case of pure compression in plane strain. Labeling the uniform stress and strain by $\bar{\sigma}$ and $\bar{\epsilon}$ respectively, we at once obtain from Hooke's laws, written for plane strain, the relation

$$
E=\left(1-\nu^{2}\right) \frac{\bar{\sigma}}{\bar{\epsilon}} .
$$

In the case $\nu=\frac{1}{3}$ which we have taken, this gives

$$
E=.888889 \frac{\bar{\sigma}}{\bar{\epsilon}},
$$

or

$$
E=.444444 \frac{\bar{F}}{\bar{a}}
$$

where $\bar{F}$ is the load required to produce the compression $2 \bar{a}$ in the simple compression test. Comparing these formulas with (4.6) and (4.7) we verify the-fact that preventing slippage in the $x_{1}$-direction has the effect of stiffening the bar. 
Given these formulas we can correct the value of $E$ determined from a test in which it is assumed that the bar is in simple compression whereas actually due to frictional end effects the bar is in a state of compression akin to that of Fig. 2. Using (4.10) one arrives at a value $\bar{E}$ for $E$ which is larger than the true value as given by (4.6). Division of these equations gives as the relation between $E$ and $\bar{E}$

$$
E=.9359 \bar{E},
$$

so that .9359 is the correction factor to be used.

It is to be remarked that in practice one can not achieve even an approximate state of plane strain in compression unless a very long (in the $x_{3}$ direction) specimen is used. However, analogous results to those given here can be obtained for a specimen with rotational symmetry. The formulas deduced for this case would be of more immediate practical value in compression testing of materials. 